\title{
Sobre los inicios de un revisionismo filosófico en Argentina y sus derivas políticas: Homero Guglielmini, Saúl Taborda y Carlos Astrada
}

\author{
On the Beginnings of a Philosophical Revisionism in \\ Argentina and its Political Commitments: Homero \\ Guglielmini, Saúl Taborda, and Carlos Astrada
}

\begin{abstract}
Resumen
El presente artículo analiza los principales textos que con distintos intereses ideológicos funcionaron como iniciadores de un particular ensayismo filosófico organizacionista y antiliberal en la Argentina desde espacios académicos y universitarios. Sus autores, Saúl Taborda (1885-1943), Homero Guglielmini (1903-1968) y Carlos Astrada (1894-1971) tuvieron una trayectoria teórica y política en buena medida común. Primero desarrollaron sus textos en términos nietzscheanos y soreleanos. Más tarde se interesaron en el romanticismo alemán y la obra de Heidegger. En este arco, desde la década de 1920 cuando estuvieron a cargo de incipientes revistas filosóficas y vanguardistas hasta el momento en que Astrada y Guglielmini se convirtieron de en dos de las más importantes plumas oficialistas del gobierno de Juan Domingo Perón, estos autores mantuvieron sus intereses anti-iluministas y anti-liberales, con los cuales se opusieron a los estudios históricos como modo de indagación. Frente a ellos, desarrollaron un ensayismo filosófico no histórico que el presente artículo periodiza y caracteriza en oposición al más estudiado revisionismo histórico. A diferencia de estos últimos, los filósofos no reaccionaron frente a las continuas olas inmigratorias, sino más bien a los proyectos políticos foráneos. Se enfocaron principalmente en la figura del gaucho, y no en la de Rosas, y propusieron una reivindicación no-católica del hispanismo. Pero, principalmente, ellos argumentaron contra el individualismo liberal y buscaron pensar herramientas teóricas a favor de un sujeto político colectivo.
\end{abstract}




\begin{abstract}
This article analyzes the theoretical and ideological interests of the first philosophical essays in Argentina from an intellectual-history perspective. Saúl Taborda (1885-1943), Homero Guglielmini (1903-1968) and Carlos Astrada (1894-1971) had similar theoretical and political trajectories. From the 1920s, when they were in charge of incipient philosophical and avantgarde magazines, they were influenced by the writings of Nietzsche and Sorel. Later, they were interested in German romanticism and Heidegger's work, in the moment when Astrada and Guglielmini became two of the most important intellectuals of Juan Domingo Perón's government. While the so-called historical revisionism has received a remarkable attention, we only have a few works on this philosophical revisionism in Argentina. It is necessary, thus, to differentiate these early revisionist writings from their counterparts dedicated to history. The philosophers did not react to the continuous waves of immigration but rather to liberal political innovations taken as "foreign ideas". They focused especially on the figure of the gaucho and proposed a non-Catholic reading of Hispanicism. In a nutshell, they argued against individualism and forth theoretical tools to think a collective subject. Therefore, this work describes a theoretical trajectory that is well known at the European level, ranging from vitalist and aestheticist irrationalism to nationalist and strongly anti-individualist organisationalist positions.
\end{abstract}

Keywords

Philosophy in Argentine, Latin American Thought, Historiography, Saúl Taborda.

\title{
Introducción. Del antiliberalismo vitalista al revisionismo filosófico: en busca de una nueva temporalidad
}

Las noticias de la Revolución rusa en Argentina llegaron poco después del inicio del primer gobierno democrático erigido con la nueva legislación electoral. Lejos de establecerse sobre un sistema político asentado, las discusiones intelectuales y culturales sobre Rusia ampliaban un nuevo ámbito de polémicas, abrían el horizonte y el menú de opciones políticas en una discusión que parecía mucho más diversa de lo esperado. Las agrupaciones estudiantiles participaron activamente de estas discusiones (Bustelo). Los profesores de filosofía más importantes de esos años tampoco quedaron afuera (Domínguez Rubio). De hecho, la necesidad de una justificación histórica y teórica de la política necesaria para el país se transformó en un tema en donde los miembros de la pequeña comunidad 
educativa en humanidades querían encontrar un espacio de intervención y consagración.

Dentro de las carreras de filosofía de Argentina en ese momento radicadas en las ciudades de Buenos Aires y La Plata, el proceso de apertura democrática se correspondió con una renovación del claustro docente, la incorporación de nuevos temas y autores en los programas de estudio y una mayor participación estudiantil del gobierno universitario a partir de 1918 (Buchbinder; Ramaglia). Al mismo tiempo, la renovación teórica ha sido continuamente caracterizada por sus propios protagonistas y la historiografía general como una "reacción antipositivista" (Alberini; Dotti). De hecho, los nuevos intereses teóricos giraron en torno a una lectura antipositivista de Kant, una relevancia primordial de la obra de Bergson, una atención discontinua a la escuela de Maburgo, una lenta llegada del neotomismo francés y una discusión constitutiva con los filósofos españoles José Ortega y Gasset (1883-1955), Miguel de Unamuno (1864-1936) y Eugenio D’Ors (1881-1954).

En el mencionado contexto de apertura política, estuvo lejos de tratarse de lecturas sin disputas y en ese momento vislumbraron lecturas teórico-ideológicas que resultan fundamentales para historizar los derroteros posteriores de sus protagonistas. En aquellos años encontramos los primeros vestigios de un catolicismo filosófico hispanista y nacionalista, vinculado a las figuras de Tomás Casares (1895-1976), Luis Guillermo Martínez Villada (1886-1959) y su discípulo Nimio de Anquín (1896-1979). También la conformación de intereses axiológicos liberales vinculados a un socialismo ético a la manera de Jean Jaurés, relacionados siempre a la figura de Alejandro Korn (1860-1936). Y la supervivencia de un socialismo cientificista filo-comunista, vinculado a la figura de José Ingenieros (1877-1925). Frente a las todavía asistemáticas lecturas de Bergson y Emilio Boutroux, muy tempranamente, en Argentina, José Ingenieros fue el primero en plantear la existencia de un vínculo directo entre el vitalismo y una política reaccionaria. 
Con esto, Ingenieros se refería a algunos jóvenes filósofos que tomaremos como protagonistas de este artículo. Según ellos, el anti-cientificismo gnoseológico y axiológico todavía era víctima de una temporalidad positivista; en algunos casos por sus compromisos marxistas con la segunda internacional, en otros casos por meros compromisos teleológicos, o quizás tan sólo por un documentalismo historiográfico empirista que consideraban excesivo. En primer lugar, dentro de este grupo de autores, destacamos a Saúl Taborda (1885-1895) y Carlos Astrada (1894-1971). Estos dos filósofos cordobeses fueron quienes dentro de la naciente historiografía filosófica desarrollada por Korn e Ingenieros a partir de 1912 quisieron romper de otro modo sus construcciones historiográficas evolucionistas. Para esto propusieron lecturas del pasado y del presente no-históricas, sino vitalistas y filo-anarquistas, encandiladas por la recién nacida experiencia bolchevique, y con referencias asistemáticas a Stirner, Nietzsche y Sorel. De hecho, en los textos del jovencísimo Carlos Astrada puede notarse que se trataba de ensayos en búsqueda de una nueva reflexión sobre la temporalidad, que su autor publicó en medios que podrían ser caracterizados dentro de lo que Doeswijk llamó “anarco-bolcheviques" (Astrada, "En esta hora que vivimos"; Astrada, "El espíritu y la historia"; Astrada, "El renacimiento del mito"; Doeswijk). Pero, como es posible observar a través de la seguidilla de revistas cordobesas que se traza a través de Mente (1920), Córdoba (1923-1925) y Clarín (1926-1927), tan sólo unos años después, sus intereses teóricos con la cultura de izquierda parecían haberse quebrado, y Sorel y Nietzsche pasaron a ser leídos únicamente en relación a las nuevas vanguardias estéticas (Bustelo y Domínguez Rubio).

En esta última dirección, unos pocos años después, desde Buenos Aires, Homero Guglielmini (1903-1968) fue otro joven lector en clave vitalista de Nietzsche y Sorel que encabezó la experiencia de la revista Inicial (1923-1927). Esta revista de vanguardia literaria también mostró inscribirse por fuera de los intereses teóricos presentes dentro de los programas universitarios y permitió a varios autores trazar sus vínculos con los primeros nacionalismos teóricos en este país (Cremonte). Resulta importante entonces incluir el derrotero de los autores que 
vamos a analizar en un marco más amplio. En Francia, Alemania e Italia, varios intelectuales habían iniciado un lento acercamiento a posiciones nacionalistas desde las vanguardias teóricas y estéticas. Los casos resultan por demás conocidos. La posición central que adoptó Giovanni Gentili en Italia tuvo un importante impacto en los intelectuales argentinos, mientras, el punto de inicio también debe ser reconocido en la amplia difusión que tuvo la obra de Oswald Spengler en los jóvenes filósofos argentinos. Por esto, cabe destacar que este tipo de indagación ensayística de análisis que vemos en la importante presencia que tuvieron en Argentina los textos de José Ortega y Gasset mantienen también este estudio spengleriano de la cultura. Como señaló Carla Galfione, sin dudas el posicionamiento discursivo de estas construcciones involucra un elitismo desde su lugar de enunciación y una crítica a los principios políticos homegeneizantes (Galfione).

En retrospectiva, Taborda, Astrada y Guglielmini, tuvieron un derrotero filosófico, ideológico y político en buena medida común. Estos tres autores abocados a la filosofía fueron parte en su juventud de lo que Martín Cremonte argumentó como un “momento soreleano”. Poco después del año 1930, su vitalismo de juventud se trocó por un existencialismo heideggeriano, al cual, dentro del ámbito filosófico, se opusieron lecturas, también germanas, pero enmarcadas en otra línea de interpretación de Husserl, provocadas por una fenomenología personalista liberal, en vínculo con los trabajos de Emmanuel Mounier y el anticomunismo francés. En la importante divisoria de discusiones entre organizacionistas-nacionalistas e individualistas-liberales de la década del treinta, paulatinamente Guglielmini y Astrada se mostraron cada vez más cerca de plataformas nacionalistas, para luego a partir de 1946 convertirse ambos en dos de los más importantes intelectuales del peronismo. En este recorrido, la discusión historiográfica y filosófica se mostró fundamental. Y la continuidad de las nociones de "mito" y "utopía" como modos de romper la experiencia histórica lineal del tiempo, en un gesto a su vez antipositivista y a la vez fuertemente politizador y nacionalista, dieron lugar al nacimiento de un ensayismo filosófico que gozó 
durante décadas de muy buena salud dentro del ámbito filosófico argentino. Con el tiempo, este ensayismo filosófico fue desarrollado desde posiciones tanto nacionalistas de derecha como de izquierda, a punto tal que muchos exponentes aseguraron que se trató del modo de indagación propio del pensamiento argentino y latinoamericano.

Las siguientes páginas historizan los inicios de este ensayismo, sus intereses teóricos y sus inscripciones políticas. Mientras, desde la historia de la historiografía argentina, el llamado revisionismo histórico ha recibido una atención notable, por su parte, sólo contamos con trabajos más bien puntuales sobre este revisionismo filosófico (Halperín Donghi).

\section{Primer revisionismo filosófico, el momento soreleano: Homero Guglielmini, Saúl Taborda y su utopía pre-capitalista}

De manera contemporánea al auge de los historiadores reconocidos como "revisionistas", y en discusión directa con otros filósofos argentinos como Alejandro Korn y Francisco Romero (1891-1962), Saúl Taborda, Homero Guglielmini y un poco más tarde Carlos Astrada dieron lugar a un ensayismo de interpretación nacional que -en principio - no ubicaba, como el resto de la historiografía, la estirpe cultural del país en la tradición de Mayo y la llamada Generación del '37. De manera que hay que situar los tópicos sobre los que giró este nuevo ensayismo filosófico en relación al arco temático que comenzaba a manejar una serie de historiadores extra-académicos ${ }^{1}$.

“QQué es la floración de librejos sobre la época de Rosas, que nada aportan, salvo los de algunos autores conocidos, y que sólo traducen audacia e ignorancia?", se preguntaba Emilio Ravignani (1886-1954) ya en 1927 desde la revista Síntesis (1927-1930) sobre estos nuevos autores. Pero específicamente fue desde 1930 cuando Carlos Ibarguren (1877-1956) — quien ya no daba clases en la cátedra 'Historia Argentina' de la Facultad de Filosofía y Letras de la Universidad de

${ }^{1}$ Para una problematización del uso del vocablo "revisionismo" para referirse a un conjunto parcialmente determinado de obras historiográficas sigo Devoto y Pagano. 
Buenos Ares y fue designado interventor de Córdoba por el gobierno de facto de José Uriburu-, junto Manuel Gálvez (1882-1962) y los principales impulsores del periódico La Nueva República (1927-1931) -José María Rosa (1906-1991), Ernesto Palacio (1900-1979)y los hermanos Rodolfo y Julo Irazusta (1897-1967 y 1899-1982) - comenzaron a especializarse en la figura de Rosas para posicionarse como opositores al liberalismo tanto de cuño anglosajón como francés. De este modo, Manuel Gálvez — quien ya en 1910 había buscado invertir la contraposición entre los dos polos establecidos como civilización (europea, racional, aristocrática, artificial) y barbarie (criolla, sensible, popular y verdadera) - apareció también como una de las figuras literarias con amplia trayectoria dentro de este grupo que conformaría la base del nacionalismo detrás del movimiento conocido como revisionismo histórico.

Antes de la formación del Instituto de investigaciones Juan Manuel de Rosas a partir de 1934, en esos años también el idealismo nacionalista de Adolfo Korn Villafañe (1894-1959) y Carlos Cossio (1903-1987) había virado hacia el antiimperialismo (Korn Villafañe; Cossio). En paralelo también se desarrolló otra serie de ensayos nacionalistas, que, si bien no estaban dirigidos a la intervención histórica, sí contribuían a plantear y discutir determinada noción del pasado argentino. Como señalaremos, en el caso de los filósofos mencionados —Guglielmini, Taborda y después Astrada-, desde giros y temas en buena medida comunes a aquellos impulsados por el revisionismo histórico, buscaron explorar formas políticas de pensar un ser nacional colectivo.

De este modo, a partir de 1930, la producción de Homero Guglielmini y Saúl Taborda antecedió a un despliegue teórico de algunas de las preocupaciones del revisionismo histórico, pero desde temas filosóficos con ciertas características y problemas propios. Si bien entonces encontramos explícitamente en ellos reivindicaciones de los caudillos populares y diatribas a favor del antimperialismo contra la oligarquía nacional, también hallamos algunas reivindicaciones del pueblo como actor colectivo, una recuperación de los elementos autóctonos y de manera mucho más mediada una argumentación a favor del desarrollo industrial y la 
intervención estatal en la economía. A lo que en cada uno de los casos y en diferentes momentos, se sumó otro de los elementos propios del nacionalismo argentino vinculado a cierta reivindicaciones hispanistas, con la particularidad de que todos estos filósofos lo hicieron desde posiciones laicas².

Todos estos nuevos ensayos partían de un diagnóstico común de crisis cultural, económica y política, ineludible tanto a partir del colapso económico como a partir del golpe de Estado de 1930. Para ellos se trataba también de una profundización de la crisis del relato liberal, tanto histórico como filosófico y cultural. De manera que surgió un replanteo entre relatos individualistas y organicistas, respecto a las cuales las experiencias europeas comenzaban a mostrar un nuevo menú de opciones políticas.

Como veremos, resulta claro que tanto Guglielmini como Taborda y especialmente Astrada no apuntaron al debate histórico. Más bien buscaron trascender este tipo de análisis en búsqueda de características constantes de la sociedad argentina, en una línea de interpretación que ellos mismos articularon a partir del Facundo de Sarmiento y en ese momento habían puesto en auge José Ortega y Gasset, Hermann Keyserling (1880-1946) y Pedro Henríquez Ureña (1884-1946). Entre 1929 y 1931, después de sus respectivas estadías en Argentina, cada uno de estos tres autores extranjeros propusieron ciertas notas comunes para caracterizar al pueblo argentino, su cultura y su comportamiento (Henríquez Ureña; Keyserling). Respecto al primero de estos tres, su visita de 1928 había dado como resultados una serie de textos cortos - "La Pampa... promesas", "Hegel y América" y "El hombre a la defensiva"- que publicó tras su regreso a España. Estos se sumaban a los Seis ensayos en busca de nuestra expresión (1928) del dominicano Henríquez Ureña editado aquí por Babel —el sello de Samuel Glusberg

2 Como veremos, estos ensayos filosóficos sobre el ser nacional escritos por Guglielmini y Taborda se inscribían en una conocida tradición local que ellos mismos filiaron con la obra de Sarmiento y Lugones. Pero además, dentro del amplio ámbito filosófico, estos autores abrieron un nuevo modo de indagación que también en la década siguiente pusieron en práctica tanto Carlos Astrada como, en menor medida, Francisco Romero. Sobre la importancia de la tradición ensayística de indagación sobre la nacionalidad en argentina y latinoamericana, ver los trabajos citados de Stabb $\mathrm{y}$ Weinberg. 
(1898-1987), que luego publicaría tanto Radiografía de la pampa (1933) como a Carlos Astrada- y que sin dudas abrieron el nuevo flanco de indagación que tras su regreso de Estados Unidos también Guglielmini comenzó a explorar (Guglielmini, Alma y estilo; Martínez Estrada).

Probablemente el caso de Guglielmini haya sido uno de los más evidentes para mostrar el recorrido ideológico-político del vitalismo esteticista al nacionalismo al que hicimos mención para luego desenvolverse como uno de los principales cuadros universitarios del peronismo. Aquel joven antipositivista redactor de la mencionada revista Inicial, después de graduarse en Filosofía y Letras, fue presidente del centro de estudiantes de la facultad porteña durante 1926, cuando se acercó por un tiempo al yrigoyenismo y comenzó su carrera docente en la Universidad Nacional del Litoral. Tuvo que dejar su puesto docente cuando al año siguiente fue el primer argentino en gozar de una beca Guggenheim con la que viajó a Estados Unidos durante un año para, tras su regreso, escribir una crónica muy crítica sobre este país (Guglielmini, Hombres entre juguetes). Ya desde estos años datan sus contactos con Homero Manzi (1907-1951) y Arturo Jauretche (19011974), quienes impulsaron la publicación de su primer ensayo de interpretación nacional titulado Alma y estilo (1930). En este ensayo Guglielmini señalaba las tensiones entre la noción de individuo y la sociedad, para atacar específicamente el utilitarismo economicista que promovía el individualismo burgués. Allí concluyó: El ser argentino significa que participo y soy ingrediente de una superestructura que me determina a su vez como argentino. Que yo sepa, muy poco se ha dicho de la esencia del alma argentina. Sin embargo, la argentinidad es un hecho que palpamos todos [...], nos gusté o no y pese a cualquier retórica internacionalista. (Alma y estilo, 146-47)

De esta manera, sostuvo que se proponía romper con lo que consideraba la línea de indagación hegemónica: el ensayo sociológico tal como lo habían desarrollado autores como Juan Agustín García, Juan B. Justo y José Ingenieros. Según Guglielmini, de distintas formas, el conjunto de estos autores habría negado los comportamientos idiosincráticos propios de la cultura argentina, como la 
"política criolla" y el "culto al coraje". Por esto, era necesario un cambio, sostuvo, no sólo por razones metodológicas, sino además por su punto de partida político; su objetivo sería posicionarse a favor de lo que Carlos Octavio Bunge había llamado "caciques hispanoamericanos" (Guglielmini, Alma y estilo).

Por esto, se proponía la tarea de descifrar aquello denostado como 'política criolla' fuera del enfoque racional y liberal que partía del individuo en un análisis político capaz de observar el "sentimiento de los pueblos". Explícitamente con esto señalaba un continuado interés por sujeto heroico del vitalismo capaz de elevarse sobre la sociedad. De modo que fue sin duda este historicismo romántico y vitalista el que le permitió tanto una crítica a la 'formalidad' y 'principismo' de los partidos políticos como una comprensión de la irrupción uriburista y los posteriores gobiernos militares.

Después de este texto inicial, Guglielmini encontraba que su ensayismo obtenía nuevos participantes exitosos en el género. Poco después de haber publicado el ensayo de Henríquez Ureña, Samuel Glusberg daba a conocer el famoso Radiografía de la pampa (1933). En 1934, desde Rosario, Astrada editó en la colección universitaria que dirigió tras su regreso de Alemania el ensayo La crisis espiritual y el ideario argentino de Saúl Taborda, quien entonces también después de 1930 hacía circular una interpretación comprensiva de la "crisis de las instituciones" y el consecuente golpe de Estado.

Tras su regreso de Alemania en 1927 y su experiencia en la dirección de la revista Clarín que heredó de Astrada, la crítica a la cultura burguesa de Taborda encontró rápidamente otros condicionantes históricos. Después del apoyo a las candidaturas de Deodoro Roca y Gregorio Bermann por el Partido Socialista en Córdoba, en 1932 fue el principal promotor de fanoe, el efímero Frente de Afirmación del Nuevo Orden Espiritual. Para este manifiesto más bien abstracto pusieron sus firmas distintos intelectuales que muy poco después tuvieron derroteros políticos bien diversos. Allí firmaron: el recién llegado Carlos Astrada, Aníbal Sánchez Reulet (1910-1998), los hermanos José Luis y Francisco Romero, Luis Juan Guerrero (1897-1957), Jorge Romero Brest (1905-1989), Luis Aznar y 
Carlos Bianchi. Con todo, la crítica común al estado de ánimo de estos pensadores no dejaba de ostentar ciertos elementos tanto antipositivistas como antimarxistas que sin embargo ahora en la nueva década aparecían trocados en relación a nuevos oponentes, principalmente el individualismo capitalista que a su vez se veía atraído por la cultura de izquierda y consumía literatura de corte realista. El diagnóstico concluía que pese a existir importantes sectores de "izquierdismo económicosocial" estos resultaban "radicalmente reaccionarios en el espíritu" (Taborda 106).

Se trataba de una crítica amplia que en un momento de reorganización político-cultural permitía a Taborda y Astrada posicionarse en Córdoba dentro del arco de las izquierdas sin adscribir a las formaciones partidarias del comunismo y el socialismo, mientras, a la luz, de sus intereses filosóficos, también de esta manera podían mostrarse en una nueva estela vanguardista, ahora teórica antes que artística. Las firmas de los hermanos Romero y de Aníbal Sánchez Reulet respondían en cambio a una diferenciación respecto a los discípulos de Korn que se afiliaban al Parido Socialista junto a su maestro; con esto, ponían en manifiesto una doble inscripción, a la vez teóricamente vanguardista, en relación al proyecto de Sur (1931-1966) que ellos tres sostenían y en contra de la filosofía marxista y la línea estética de la revista Claridad (1926-1941) como plataforma cultural y editorial del Partido Socialista.

De hecho, fue en última misma revista, Claridad, donde Alberto May Zubiría a partir de este manifiesto acusó especialmente a Taborda y a Astrada de estar detrás del proyecto de una nueva organización "fascista" llamada "Joven Argentina" de la cual los firmantes de fanoe constituirían su comité consultivo. May Zubiría era uno de los militantes socialistas atento tanto al movimiento estudiantil como a criticar el naciente nacionalismo historiográfico dentro del cual ubicaba este "manifiesto inicial fascistizante" firmado por "revolucionarios espirituales y filósofos que acaudillan Saúl Taborda y Carlos Astrada... declarados hoy fascistas rabiosos" (May Zubiría). Como veremos, desde esta temprana crítica de 1933, con el correr de los años tanto Taborda como Astrada resultaron 
especialmente sospechosos para el movimiento estudiantil, quienes los catalogaron como nacionalistas, fascistas y/o nazis.

Con esta crítica a cuestas, Taborda insistió al año siguiente de fanoe con su diagnóstico de "crisis espiritual”. Como dijimos, Carlos Astrada publicó desde su Instituto Social de la Universidad Nacional de Litoral la nueva obra de Saúl Taborda, titulada precisamente La crisis espiritual y el ideario argentino (1934). Este nuevo texto de Taborda mantenía una concepción libertaria negativa del Estado que sin embargo ahora aparecía en buena medida matizada como posible herramienta contra el imperialismo ${ }^{3}$. Sin embargo, si esta es la causa de la "crisis espiritual", como sostenía desde el título del libro, la solución parecía estar latente en el propio "ideario argentino", aunque éste no se encuentre de hecho en las ideas, sino oculto y deba ser revelado.

De modo que era aquí donde incluía una crítica común a los intelectuales, a los universitarios y a la izquierda, que, en todos los casos, pensaba totalmente alejados, no sólo de la "realidad", sino además desconociendo la tradición política efectiva del territorio argentino. Con esto, además, Taborda se alejaba directamente de la filosofía profesionalizada que proponía el en ese momento el filósofo y decano de la Universidad de Buenos Aires Coriolano Alberini (1886-1960). Muy lejos de la universidad, según Taborda, la filosofía de aquel momento se encontraba totalmente alejada de "la sociedad viviente" y de "los afanes de los talleres y los campos". De la misma manera, respecto a la izquierda entonces, mientras el Partido Radical había podido mover gran cantidad de sufragios, el Partido Socialista, sin posibilidad de éxito, sólo servía para otorgar acreditaciones de interés político para

3 Tras su regreso de Europa en 1926, Saúl Taborda no volvió a ejercer como profesor. Se hizo cargo de la dirección de Clarín (1926-1927) y participó de la filial cordobesa de la Unión Latinoamericana. De allí en más, sin volver a enrolarse en ninguna institución educativa, publicó la totalidad de sus escritos posteriores en revistas de filosofía, en editoriales universitarias o en su propia revista Facundo (1934-1939). Sobre sus adscripciones filosóficas, en estos textos Taborda realizó referencias tanto a la axiología como a la fenomenología y al existencialismo, utilizando o criticando aspectos puntuales, pero sin dedicarse específicamente a inscribir sus indagaciones en alguno de estos enfoques filosóficos. 
los intelectuales. Éstas constituían las coordenadas que según Taborda generaron un irracionalismo vitalista cuya "crisis espiritual" supo aprovechar el fascismo.

Con esto, en 1930 señalaba el divorcio efectivo no sólo entre universidad y pueblo, sino entre los "intelectuales y el pueblo". Según Taborda, la Reforma universitaria había apuntado a romper este status quo, pero no había discutido la calidad de un determinado tipo de ideas históricamente hegemónicas. Contra esto, Taborda indicó que la solución política propuesta debía resultar acorde a las características constitutivas de la sociabilidad argentina presente en las costumbres provinciales, basadas en un pasado a la vez gauchesco, comunalista, caudillesco e hispánico, que en buena medida se combinaban en lo que continuamente Taborda bautizó "lo facúndico". De esto modo, a cien años del fallecimiento del caudillo riojano Facundo Quiroga, Saúl Taborda lanzó su revista Facundo (1935-1939) donde proponía esta nueva palabra — "facúndico"- con el fin de otorgarle características positivas de tinte tanto nacionalistas como comunitaristas, a las que reconocía raíces socialistas y anarquistas ${ }^{4}$.

Es de destacar entonces que -al igual que lo hará Astrada diez años después- la genealogía política construida por Taborda carece de nombres propios, ideas y en general de cualquier elemento textual e incluso contextual. Si bien no lo dice, podría pensarse como mito, aunque —como había deslizado asistemáticamente Guglielmini y Astrada sostuvo poco después- parece pensar estas distintas características, como existenciarios heideggerianos, ocultos por una pésima importación que se dio a partir de la colonización: "colonización que hoy, a la luz de lo facúndico, consideramos inadecuada". Por esto, sostuvo Taborda, para encontrar al "hombre-entero" o Dasein completo argentino, al modo del existencial

$4 \mathrm{La}$ construcción tabordiana alrededor de la figura de Facundo Quiroga no es desarrollada de manera clara ni mucho menos. En líneas generales, su pasó por la historia quedó registrada como la del "caudillo de caudillos" y con esto la máxima expresión de autonomía respecto a Buenos Aires: "Facundo era nuestro héroe. Encarnaba en modo admirable ese fondo de heroísmo que construye los pueblos y les imprime su sello de inmortalidad. [...] Es la expresión más alta de la vida comunal, la perfecta relación de la sociedad y el individuo, concertada por el genio nativo...". 'Facundo' es entonces en definitiva sinónimo de federalismo, pero en el tono de mito útil que aparecerá de manera similar fragmentariamente en Astrada en los años siguientes, pero principalmente de manera más sistemática a partir de 1946. Ver: Taborda, La crisis espiritual. 
imposible de Heidegger, hay que pensar entonces al "hombre pre-capitalista", que en el caso argentino estaría dado en el hombre pre-liberal. Dice Taborda, para, sobre esas notas distintivas, crear instituciones "originales", expresivas de la “idiosincrasia nativa". Según él, entonces, la "Voluntad de Mayo" no estaba representada en el liberalismo cosmopolita sino en el comunalismo personificado en Facundo, el cabildo como núcleo de la representación popular y la tradición hispanista (Taborda 15).

Con todo, ese pasado comunal se vuelve casi mítico, sin mayores determinaciones explícitas y únicamente representado como última instancia en un Facundo Quiroga ahistórico. La narración se vuelve sumamente imprecisa. Traza la transformación del nomadismo al sedentarismo argentino en espacios urbanos bajo fines de supervivencia militares y políticos posibilitados por el poder de un caudillo. El mestizaje español de alguna manera se acopla a este formato tribal, sumándole una épica feudal y caballeresca. En esta semblanza, el ingreso de la cultura letrada trajo para Taborda el comienzo, no sólo de la mentira y la falsedad, sino también la caída en la historia, cuando comenzó la voluntad de forzar un modelo político de manera sangrienta y por eso, para Taborda, condenado a carecer de éxito social. Este esencialismo no critica ahora directamente la religión como fenómeno social sino específicamente el modo que asume en la Iglesia católica como unificadora de esas prácticas.

Veremos que fue Astrada y luego una larga tradición aquella que recogió varios gestos de estos ensayos de Taborda. Poco después, Guglielmini también fue parte del "giro telúrico" dentro del ensayismo que se dio a partir de la obra de Taborda y la Radiografía de la pampa. Pero, en retrospectiva su caso no podría ser tan retomado ni citado como antecedente por su participación directa dentro de los cuadros nacionalistas y los sucesivos cargos de gestión que ocupó a lo largo de varios gobiernos militares a partir de 1935, cuando se hizo cargo de la Comisión Nacional de Cultura junto al mencionado Carlos Ibarguren. Mientras, de hecho, Guglielmini escribió entre 1941 y 1944 en las revistas nacionalistas Choque (19431943) y Cabildo (1943-1944). De estos años dató una importante cantidad de libros 
ensayísticos de claro apoyo al golpe militar del 43: La comisión Nacional de Cultura y sus fines (1943), El oro y la espada (1943) y El presidente Castillo y la afirmación de la soberanía nacional (1943). Como indican sus títulos mediante los que justificaba directamente el golpe de Estado de 1943 antes de ocupar importantes posiciones durante el peronismo, cuando también volvió a ocupar un cargo docente, como titular de 'Historia de la literatura argentina' en el momento en que Ricardo Rojas era obligado a renunciar. De allí en más, Guglielmini trazó su propio canon nacional, que comenzaba en una lectura invertida de El Facundo de Sarmiento, seguía con El Payador (1916) y una importante valoración de la obra tardía de Lugones y continuaba con el género de textos que después Astrada compilaría en El mito gaucho (1948), en donde también implícitamente incluís con su propia obra. La diferencia específica por la cual se destacaba su obra y la de Astrada sobre la de Sarmiento y Lugones se debía, según Guglielmini, a que resultaban afirmativos y no únicamente nostálgicos, aporéticos y disolutivos.

\section{Segundo revisionismo filosófico, el momento heideggereano: Carlos Astrada, mito, nación y antiliberalismo}

Después de su regreso de Alemania, todo el grupo de filósofos amigos conformado por Taborda, Astrada, Guerrero y Alberini desarrolló un interés permanente por la filosofía existencial alemana. Por su parte, la otra figura en ascenso, el militar-filósofo Francisco Romero apadrinado por Alejandro Korn, también se mostró pendiente durante décadas de la nueva meca filosófica que tenía la Argentina, aunque Romero escogió otros pensadores germanos respecto a los cuales referir su obra. En general Alberini y F. Romero mantuvieron un distanciamiento crítico al vitalismo, mientras Guglielmini y Astrada mostraron mayores continuidades.

Específicamente Astrada, tras su regreso de Alemania en 1932, buscó publicar su primer libro titulado El juego metafísico en la editorial Babel de Samuel 
Glusberg, en el mismo año en que éste publicó Radiografía de la pampa $(1933)^{5}$. Al leer este último, Astrada felicitó a Glusberg y sostuvo que a él también le interesaba "la existencia pampeana":

Leí Radiografía de la pampa. Muy buena. Mañana o pasado le escribiré a Martínez Estrada. Hace tiempo que me preocupa el tema de la existencia pampeana. Medito un esquema desde el punto de vista existencial. El estudio histórico-sociológico de Estrada es un valioso aporte a la elucidación del problema desde otro punto de vista o en otra zona ${ }^{6}$.

Sin embargo, todavía tardó unos cuantos años más en publicar sus primeros ensayos de interpretación nacional y proponer él también su propia exploración literaria y existencial a partir de 1939 con la aparición de los diferentes ensayos que más tarde darían lugar a El mito gaucho (1948). Por un lado, durante esos años publicó sus artículos filosóficos en revistas universitarias como la Revista de la Facultad de Ciencias Económicas de Rosario, Humanidades y Revista del profesorado, o en revistas culturales como Trapalanda, Cursos y Conferencias, Nosotros y Sur —en esta última sólo hasta 1939, cuando las diferencias con su grupo editor se hicieron más evidentes. Por otro lado, comenzó a publicar otra serie de textos ensayísticos, ontológicos y literarios en buena medida paralelos en

5 Según él mismo, Nimio de Anquín y Luis Guillermo Martínez Villada - los cordobeses que encabezaban el neo-tomismo fascista argentino - impedían su ingreso a la Universidad Nacional de Córdoba. Frente a esto, llegó a pensar incluso en aceptar la dirección de un reformatorio de menores en Rosario pero pudo conseguir un mejor puesto como Director del Instituto Social en la Universidad del Litoral en esa misma ciudad, desde donde editó la mencionada Crisis espiritual... de Taborda y un folleto de Francisco Romero sobre la filosofía de la cultura. Allí permaneció durante tres años hasta ingresar en 1936 como profesor adjunto de 'Historia de la filosofía (II)' en la Facultad de Filosofía y Letras de la UBA. En 1937 comenzó a enseñar también 'Ética' en la Facultad de Humanidades y Ciencias de la Educación de la Universidad Nacional de La Plata, cargo que ocupó hasta 1946. En 1939 obtuvo cargos como profesor de 'Filosofía', 'Lógica' y 'Psicología' en el Colegio Nacional de Buenos Aires, que mantuvo hasta 1948. En este año renunció a todas sus cátedras cuando consiguió el puesto como profesor titular de 'Gnoseología y metafísica’. Gracias a este cargo en 1950 logró el primer puesto con dedicación exclusiva de FFYLUBA que ocupó hasta 1956 cuando lo obligaron a concursarlo nuevamente y se rehúso a ello.

6 Carta de Astrada a Samuel Glusberg fechada el 06/08/1933 - Fondo Samuel Glusberg (FSG 1.71/ S.5) - CEDINCI. 
revistas culturales orientadas a una literatura nacionalista, en donde publicó su texto de tono programático "Para una metafísica de la pampa". En esta segunda dirección, uno de los primeros textos en donde Astrada exploró el ensayismo sobre el ser nacional había sido "Meditación de Rumipal” (1939) al que se sumó en 1943 su texto rilkeano "El encargo de la tierra" publicado en su compilación de ensayos Temporalidad (1943). A estos se sumaron dos textos suyos que se convirtieron en el principal foco de ataque de los estudiantes comunistas.

Uno de ellos fue un breve texto publicado en la revista institucional Estudios germánicos dirigida por el novecentista Juan Probst. Aquí Astrada sostenía que la indudable actualidad del ya discutido Martin Heidegger se debía a un "clima existencialista" previo que había llegado a ser vislumbrado por Kierkegaard, Unamuno y Rilke, a quien aquí admite seguir en sus ensayos "telúricos”, capaces de, como el "misticismo cristiano", lograr intuiciones de "consecuencias inéditas". Este texto fue continuado por el mencionado texto programático "Para una metafísica de la pampa" publicado en la revista literaria Antología (1944-1945) dirigida el poeta nacionalista Arturo Cambours Ocampo (1908-1996). En los cuatro números que duró esta revista vinculada a las editoriales que seguían funcionando en Madrid después de la Guerra Civil Española, Astrada compartió firmas con un particular elenco nacionalista formado por Carlos Ibarguren, Leopoldo Marechal (1900-1970), Tomás D. Casares, Homero Guglielmini, Ignacio B. Anzoátegui (1905-1978), Octavio Nicolás Derisi (1907-2002) y José María Rosa (1906-1991).

En este texto propositivo que abría oficialmente la indagación que daría lugar unos años después a El mito gaucho (1948), Astrada sostenía heideggerianamente que el hombre argentino poseía una condición de especial apertura hacia el ser que se manifestaba en una gran angustia ontológica. Esta melancolía se debía especialmente a su doble carácter de eyectado, por su humanidad misma y por su falta de una cultura propia que se manifestaba en el acto de habitar la pampa; es decir, en palabras del anterior texto de Taborda, por la "colonización inadecuada" que había sufrido. Por eso, el "ser argentino", antes que nadie, se debía su propio análisis existenciario, que, dice Astrada, nada tiene que 
ver con la tarea histórica. Como había intentado más de veinte años antes, Astrada pretendió con otras herramientas y referencias romper con un análisis histórico evolucionista.

En un ambiente cultural donde la segunda gran guerra generaba un fuerte anti germanismo especialmente presente en los círculos izquierdistas, con estos textos, en los primeros años de la década del cuarenta, Carlos Astrada se transformó en la figura más polémica de toda la Facultad de Filosofía y Letras de la Universidad de Buenos Aires. Profesor adjunto de 'Historia de la Filosofía' y miembro del Consejo Directivo desde ese mismo año, fue uno de los que se quejó de la creciente politización universitaria que especialmente apuntaba contra su figura.

Al menos desde 1942 Carlos Astrada fue sistemáticamente acusado de “filósofo oficial nazi”. La juventud comunista de la Facultad no sólo pegó afiches en las paredes contra Carlos Astrada, sino que le dedicaron numerosos artículos desde las revistas Orientación (1936-1949) y más tarde desde Nueva Era (19491976), escritos principalmente a cargo de los militantes Isidoro Flaumbaum (1942), Berta Perelstein (1913-2008) y Ernesto Giudici (1907-2015). Su crítica radicaba en las elecciones filosóficas mismas, germanistas, irracionalistas y oscurantistas. Criticaban también el mismo tono de su lenguaje, y el vocabulario impreciso con palabras como "sangre", "patria” y "suelo", que relacionaban directamente a la idea de "raza" presente en Mein Kampf(1925).

Según los estudiantes, Astrada era uno de los directores-fundadores del período nacionalista Choque (1942-1943) junto a Lizardo Zía —quien aparecía como único director responsable- y del cual también participó Guglielmini ${ }^{7}$. La confirmación de esta participación para ellos habilitaba por sí sola la destitución de

7 Cabe destacar que en este contexto Astrada compiló y editó sus textos de juventud bajo el título Temporalidad (1943) sin incluir sus textos libertarios y filo-bolcheviques a los que hicimos mención la primera parte de este artículo. Con esto, en su propia construcción autobiográfica Astrada negó ese período inicial refiriéndose a sus escritos reformistas como su "período literario", en momentos en que participaba de una intelectualidad nacionalista opuesta a la radicalización estudiantil. 
Astrada como profesor de la universidad. Lo acusaban también de ser antisemita y de tener una "esposa nazi”. Sin embargo, su principal argumento consistía en que el heideggerianismo y la filosofía alemana en general involucraba una posición nazi por la sola implicancia de sus ideas. Para lograr caracterizarlo aún más cercano a la derecha, a esto sumaban que su "padrino" filosófico local era Coriolano Alberini, a quien llamaban de hecho "Dr. Nazi". Claro que tampoco le perdonaban su lectura no doctrinaria de Marx que hasta hace pocos años nunca había dejado de abordar en sus artículos.

Por esas mismas fechas, el militante Isidoro Flaumbaun y el futuro filósofo analítico Hernán Rodríguez publicaron también en Orientación un análisis sobre los estudios filosóficos. Básicamente criticaban la hegemonía que tenían allí los pensadores alemanes. Y, en el contexto de la Unión Democrática, para ellos esto constituía una contradicción en la loable tarea contra la "barbarie nazi" que llevaba a cabo Francisco Romero. Según estos estudiantes, a diferencia de Astrada, F. Romero estaba bien ubicado, pero no veía el problema fundamental de estar embarcado en la filosofía alemana (Flaumbaum y Rodríguez). Como veremos, se trata de una crítica al irracionalismo filosófico que todavía no aparece dentro de los estudios filosóficos y sólo será desarrollada de manera sistemática por la revista Minerva (1944-1945) de Mario Bunge ${ }^{8}$.

En retrospectiva, Carlos Astrada justificó metodológicamente sus ensayos en varios momentos y de diferentes maneras, en ninguno de los casos muy

8 En concreto, no hay constancia de colaboraciones suyas en el periodismo nacionalista de esos momentos hasta su texto "Para una metafísica de la Pampa" publicada en la mencionada revista Antología. Pero, como señalamos, los estudiantes del Partido Comunista lo acusaban de publicar artículos sin firma. Se trata de un período del pensamiento de Carlos Astrada del cual aún no se ha recuperado su bibliografía completa; aparentemente ni sus discípulos y ni sus biógrafos han querido hacerlo. Sin embargo, según Enrique Zuleta Álvarez, su militancia nacionalista quedó registrada en su participación en el Congreso Nacionalista realizado en Buenos Aires el 16 de diciembre de 1942 en donde estuvieron, entre otros, Nimio de Anquín, Ramón Doll, César Pico y Vicente Sierra. Respecto a estos referentes, Zuleta Álvarez — con un conocimiento interno de la causa- lo señaló, junto a Scalabrini Ortiz, entre los escasos militantes nacionalistas no católicos. De esta manera, a diferencia de sus colegas filósofos que quedaron dentro de la facultad tras la eclosión universitaria de 1946, Astrada y Guglielmini contaban con una militancia nacionalista previa. También desde otra posición Juan A. Vázquez relató el entusiasmo de Astrada durante los años en que las potencias del Eje parecían en camino a ganar la Segunda Guerra Mundial. Ver los trabajos citados de Vázquez y Zuleta Álvarez. 
concretamente. Durante la década del treinta, mediante estos ensayos Astrada (1936) insinuaba explorar una asistemática analítica existencial al modo de Ser y tiempo, pero con marcados elementos "telúricos" del romanticismo alemán. Sin embargo, en 1947, Astrada pareció indicar que se trataba de una indagación interesada en la antropología en sentido pragmático de corte kantiano. Con todo, sin dudas, a pesar de estas indicaciones marginales, la opción más recurrente fue una interpretación del Martín Fierro en forma de mito popular a la manera soreliana en busca de un modo de activación política. Recordemos que en 1921, frente a las fuertes críticas al proceso soviético que se esbozaban desde muchos sectores anarquistas contra las plataformas de las que él participaba, también Astrada propuso una instalación mítica de la Revolución rusa como motor de cambio más allá de la evaluación concreta del proceso.

\section{A modo de epílogo. En las puertas del tercer ensayismo filosófico: derivas nacionalistas y decoloniales, el momento hegeliano}

Las páginas anteriores identificaron un grupo de varones abocados a la filosofía que ocuparon importantes cargos de gestión universitaria e impulsaron las principales revistas e instituciones filosóficas durante los años abordados. Su marca en común es haber desarrollado un ensayo identitario de la nacionalidad argentina vinculado a los estudios filosóficos universitarios; primero en términos nietzscheanos y soreleanos, después interesados en el romanticismo alemán y la obra de Heidegger, y, finalmente, en un tercer momento a partir de 1950, enfocados en Hegel. Siempre, en todos los casos, a través de las décadas, con objetivos anti iluministas, antiliberales y antihistóricos.

Mientras los historiadores revisionistas que surgieron a partir del Instituto de Investigaciones Juan Manuel de Rosas recibieron una atención constante, sus contrapartes filósofos apenas han recibido consideraciones en relación a su tarea de construir una imagen del pasado en términos filosóficos. Sobre los historiadores, Tulio Halperín Donghi, sostuvo que sus textos consistían más bien en "lecciones 
culturales”. Mientras, Terán se refirió a estos ensayos de interpretación nacional de Taborda y Astrada como “ontológicos” y "a-históricos” (Halperin Donghi, Terán).

Con todo, se trataba entonces de una defensa de la tradición hispano-criolla que en el caso de los filósofos mostraba importantes diferencias con la mayoría de las principales obras del revisionismo histórico ${ }^{9}$. En primer lugar, estos no reaccionaban a las continuas oleadas inmigratorias sino más bien frente las innovaciones políticas liberales tomadas como "ideas foráneas". En segundo lugar, queda claro que se centraban especialmente en la figura del gaucho y los caudillos $-\mathrm{y}$ no en la de Rosas-, por lo que en todo caso vislumbraban un nacionalismo no tan aristocrizante como el de algunos historiadores revisionistas. En tercer lugar, a contramano del nacionalismo revisionista, estos autores propusieron una lectura no católica del hispanismo, que recuperaba sobre todo un hipotético nexo hispánico con los elementos de origen greco-latino como había hecho Lugones, por ejemplo, en el El payador. En cuarto lugar, también los giros anti mercantilistas siempre anti anglosajones que hacían mención a Estados Unidos o a Inglaterra como foco del imperialismo político y económico aparecían siempre de manera mucho más mediada a partir de una preocupación principalmente dirigida al sistema político. Fundamentalmente, para esto se propuso apuntar contra el individualismo pensando un sujeto colectivo, ya sea con la figura de Facundo o con la figura del gaucho. De modo que, así como el revisionismo se planteó como una "contra historia", este ensayismo filo-filosófico se planteó como un contra filosofía del sujeto político liberal fundado en la razón ${ }^{10}$.

9 Habitualmente se han distinguido dos núcleos, uno antiimperialista, rosista y católico, con referentes como Julio Irazusta, Vicente Sierra, José María Rosa y Ernesto Palacio. Otro también antiimperialista e yrigoyenista conformado por Raúl Scalabrini Ortiz y Arturo Jauretche. Ver por ejemplo Devoto y Pagano.

10 En menor medida, también existió una indagación ensayística liberal a partir de la obra de Eduardo Mallea y Francisco Romero que se desenvolvió siempre cercana a la revista Sur y de la cual Héctor P. Murena (1954) fue el principal continuador después de 1955 desde las filas de la Asociación por la libertad de la Cultura. Ver: Mallea, Historia de una pasión argentina; Romero, "La realidad espiritual argentina", "Meditación de Occidente", Murena, El pecado original de América. 
Se trataba entonces de una nueva respuesta a la demanda romántica realizada la filosofía y a la historia bajo el lema de "fortalecer de la conciencia nacional". Pero mientras Ingenieros, Korn y Alberini reconocían estrategias para su escasa difusión y apuntaban de distintas maneras a las "minorías", Taborda y Astrada, al menos problematizando esto en algunas de sus obras, tomaron para esta tarea un género más cercano a las indagaciones ensayísticas. Como conclusión, podemos decir que, si bien no había una interacción directa entre ambos géneros, estas construcciones de Taborda, Astrada y Guglielmini consistieron en una indagación esencialista alejada del esquema evolutivo y progresista de las historias del pensamiento argentino que habían desarrollado Korn e Ingenieros y que en esos años también mantenía Alberini ${ }^{11}$.

Esquemáticamente se trata entonces de dos concepciones de la historia. Una evolucionista - ya sea de corte spenceriano, marxista, jaureseano o croceano. Frente a otra identitaria y ensayística - ya sea al modo soreliano, nietzscheano o heideggeriano. Su apuesta es fuerte: la historia no involucra conocimiento histórico. La filosofía y sus nuevos métodos de indagación pueden lograr mayor conocimiento histórico. Del mismo modo se trata de distintas concepciones del tiempo. Un tiempo histórico. Y un tiempo filosófico. El segundo resulta necesariamente colectivo: el individuo sólo se descubre a posteriori e intuye su participación de un acontecer orgánico. En cambio, el tiempo histórico parece ubicarse bajo las categorías necesarias de un antes y un después que necesariamente involucra un trabajo de concientizar estos momentos a nivel individual, respecto a los cuáles no sólo se organiza la historia sino también la sociedad y su desarrollo. Desde el punto de vista no evolucionista, la identidad nacional no redunda en una construcción — todavía en curso respecto a la cual la propia narración contribuyesino bajo la forma de un descubrimiento de los elementos idiosincráticos. El primero de ellos con un gesto constructivista de identificación y propagación de los elementos útiles para esta tarea. El segundo con una intencionalidad netamente

11 Korn, Influencias filosóficas en la evolución nacional; Ingenieros, La evolución de las ideas argentinas; Alberini, Problemas de la historia de las ideas. 
romántica que no construye, sino que descubre la nacionalidad. De alguna manera, marcan dos registros sobre la geografía. Una que la considera ya de por sí parte de la historia, sólo como una materia extensa científica, para ser ordenada e industrializada. Otra como necesariamente abierta que encubre una interpretación.

De alguna manera, este viraje debía ser acompañado por una versión decadentista del avance histórico que se llevaba puesto al mismo tiempo al conocimiento histórico. La "evolución" - ya sea nacional, de la historia o de las ideas- no sólo obtuvo algunos avisos antipositivistas, que habría que rastrear quizás en el mito sorealiano del sindicalismo revolucionario argentino, quizás en la traducción de 1924 del texto de Simmel por parte de Astrada, quizás en la lectura de Spengler sobre la cual llamó la atención Ernesto Quesada y respecto a la cual alertó un joven León Dujovne, quizás en las lecturas de Maurras —cuya importancia Halperín remarcó para el revisionismo católico pero fue poco frecuentada por los filósofos locales-, o quizás mejor en la búsqueda de existenciarios propuesta por el mismo Heidegger.

Sin dudas, esta pregunta nos lleva más bien a pensar la importancia de un best-seller como La decadencia de occidente —cuya versión definitiva de 1922 tuvo numerosas reediciones por Espasa-Calpe- y el rechazo del texto por Francisco Romero. Pero sobre todo nos lleva al recorrido astradeano, que fue de "El renacimiento del mito" publicado en la revista anarco-bolchevique Cuasimodo en 1921, pasó por el texto traducido y publicado de Simmel en 1924, hasta llegar a “La deshumanización de occidente". Pero el objetivo explícito, y más tarde muy perdurable, de estos ensayos fue lograr una indagación que no quiere volver a “importar" conceptos que considera ajenos, sino comenzar a explorar cuáles serían los elementos "originarios" a tener en cuenta: el humanismo español, su mística, el caudillismo, la organización comunal federativa...

Finalmente, éste fue el modo de indagación que se instaló como "propiamente latinoamericano" tras la llegada de las nuevas obras del mexicano Leopoldo Zea y las discusiones del Primer Congreso de Filosofía de 1949. A partir de este momento, el ensayismo filosófico cobró un éxito notable. En este artículo 
buscamos identificar los primeros ensayos parte de este género filosófico que sin duda tendría su apogeo durante los años del gobierno del Juan Domingo Perón, cuando, según Devoto y Pagano, los debates historiográficos con el revisionismo alcanzaron una "intensidad y virulencia que no tuvo parangón en ningún otro país de Iberoamérica" (Devoto y Pagano). Si bien a partir de ese momento también las críticas a este ensayismo filosófico "irracionalista" ya eran conocidas, a partir de ellos se estableció una verdadera tradición de la "descripción de la conciencia americana", fundamentalmente por parte de Astrada, Nimio de Anquín y Rodolfo Kusch, a quienes de a poco se sumarían también desde diferentes posiciones políticas Héctor Murena, Arturo Roig y Enrique Dussel. De hecho, este ensayo filosófico logró una amplia difusión y distintas inscripciones políticas, que fueron desde el nacionalismo y el peronismo de derecha hasta el ensayismo liberal y los desarrollos de una teología descolonizadora. Muchos incluso sostuvieron que se trató del género propio de la filosofía latinoamericana. Y, en casi todos los casos, los mencionados textos de Taborda y Astrada, respectivamente La crisis espiritual y El mito gaucho, resultaron una referencia obligada al momento de reconocer a los iniciadores de este género. En cambio, seguramente por sus vínculos a distintos gobiernos militares, la figura de Guglielmini no ha sido recuperada como tal.

\section{Bibliografía}

Alberini, Coriolano. Problemas de la historia de las ideas filosóficas en la Argentina. Instituto de Estudios Sociales y del Pensamiento Argentino, Universidad Nacional de la Plata, 1966.

Astrada, Carlos. "El clima de la filosofía existencial”. Estudios germánicos, vol. 8, 1942 de 1941, pp. 90-94.

. "El espíritu y la historia". Mente: revista de crítica social, núm. 2, junio de 1920, pp. 4-6. 
.El mito gaucho. Cruz del sur, 1948.

. "El renacimiento del mito". Cuasimodo, núm. 20, junio de 1921, pp. 1-

2.

. "En esta hora que vivimos". Revista del Centro de Estudiantes de

Derecho, núm. 1, agosto de de 1919, pp. 6-13.

. "Fetichismo constitucional". Reforma de la constitución argentina, Universidad de Buenos Aires. Acción Social Universitaria, 1948.

. Goethe y el panteismo spinoziano. Universidad Nacional del Litoral, 1933.

. "Meditación de Rumipal”. Revista del profesorado, octubre de 1939.

. "Surge el hombre argentino con fisonomía propia". Argentina en

marcha, Comisión Nacional de Cooperación Intelectual, 1947.

. Temporalidad. Cultura Viva, 1943.

Astrada, Carlo, Carta a Samuel Glusberg, 06/08/1933, Fondo Samuel Glusberg (fsg 1.71/ S.5) - Centro de Docmentación e Investigación de la Cultura de Izquierdas - Universidad Nacional de San Martín.

Buchbinder, Pablo. Historia de la Facultad de Filosofía y Letras: Universidad de Buenos Aires. 1a ed, Editorial Universitaria de Buenos Aires, 1997.

Bustelo, Natalia. La reforma universitaria desde sus grupos y revistas: Una reconstrucción de los proyectos y las disputas del movimiento estudiantil porteño de las primeras décadas del siglo XX (1914-1928). Universidad Nacional de La Plata, 2016, http://www.memoria.fahce.unlp.edu.ar/tesis/te.1307/te.1307.pdf.

Bustelo, Natalia, y Lucas Domínguez Rubio. "Vitalismo libertario y Reforma Universitaria en el joven Carlos Astrada”. Políticas de la memoria, vol. 16, 2015, pp. 295-310, doi:10.15446/achsc.v44n2.64014.

Cossio, Carlos. La revolución del 6 de septiembre: introducción filosófica a su historia y esquema universal de la política argentina. La Facultad, 1933.

Cremonte, Martín. El momento soreliano. Vitalismo, juvenilismo y fascismo en la revista Inicial (1923-1927). Universidad Nacional de San Martín, 2019. 
David, Guillermo. Carlos Astrada: La Filosofía Argentina. El Cielo por Asalto, 2004.

Devoto, Fernando, y Nora Pagano. Historia de la historiografía argentina. 1a ed, Sudamericana, 2009.

Doeswijk, Andreas. Los anarcobolcheviques riolatenses (1917-1930). CeDInCI, 2013.

Domínguez Rubio, Lucas. "Filosofía e historia en las primeras historias de las ideas argentinas: la discusión historiográfica entre José Ingenieros y Alejandro Korn”. Prismas: revista de historia intelectual, vol. 21, 2017, pp. 75-94.

Dotti, Jorge Eugenio. La letra gótica: recepción de Kant en Argentina, desde el romanticismo hasta el treinta. Universidad de Buenos Aires, Facultad de Filosofía y Letras, 1992.

Fernandez, Maria Angela. Limen de la historia del pensamiento y cultura argentinos. Talleres graficos pedemonte, 1955.

Flaumbaum, Isidoro. "Filosofía y filósofos del campo de la guerra". Nueva Era, 1949, pp. 658-779.

Galfione, María Carla Galfione. "Filosofía y política en los orígenes. Lecturas de Ortega y Gasset”. Anacronismo e irrupción: Revista de teoría y filosofía política clásica y moderno, vol. 8, núm. 14, 2018, pp. 209-33. . "La revolución como presente: Filosofía y política en la Revista de Filosofía”. Prismas: revista de historia intelectual, vol. 21, 2017, pp. $233-39$.

Guglielmini, Homero, et al. Argentina en marcha. Comisión Nacional de Cooperación Intelectual, 1947. . "La formación de una conciencia técnica nacional”. Antología, vol. 2, 1945, pp. 4-7.

Guglielmini, Homero Mario. Alma y estilo. Gleizer, 1930. . Hombres entre juguetes. Anaconda, 1933. . Temas existenciales. Losada, 1939. 
Halperín Donghi, Tulio. Ensayos de historiografía. 1a ed, El cielo por asalto, 1996.

Henríquez Ureña, Pedro. Seis ensayos en busca de nuestra expresión. 1a ed, Babel, 1927.

Ingenieros, José. La evolución de las ideas argentinas. L. J. Rosso, 1937.

Keyserling, Hermann. Meditaciones sudamericanas. Espasa calpe, 1933.

Korn, Alejandro. La libertad creadora. Claridad, 1936.

Korn Villafañe, Adolfo, editor. Unitarios y federales: ética, estética y técnica del Estado Argentino. Imprenta de la Universidad, 1931.

Kusch, Rodolfo. "Inteligencia y barbarie”. Contorno, vol. 3, 1954. . La ciudad mestiza. Quetzal, 1952. . La seducción de la barbarie. Raigal, 1953.

Mallea, Eduardo. Historia de una pasión argentina. Anacondo, 1938.

Martínez Estrada, Ezequiel. Radiografía de la pampa. Babel, 1933.

Perón, Juan Domingo, et al. Tribuna de la revolución: conferencias. Editado por Carlos Astrada, Nueva Argentina, 1948.

Ramaglia, Dante. “Condiciones y límites del proceso de institucionalización de la cultura filosófica argentina a comienzos del siglo XX”. Solar, vol. 6, 2010, pp. 13-39.

Ravignani, Emilio. “Los estudios históricos en Argentina”. Síntesis: artes, ciencias y letras, vol. 1, 1927, pp. 51-76.

Rodeiro, Matías. "Derivas de Saúl Taborda por los afluentes estéticos de la Reforma Universitaria, entre el modernismo y las vanguardias, 19091927”. La Biblioteca: revista fundada por Paul Groussac, vol. 14, 2013, pp. 427-63. . “Introducción”. Escritos políticos (1918-1934), BN-UNC, 2008.

Romero, Francisco. "La realidad espiritual argentina". Realidad: revista de ideas, vol. 4, 1947, pp. 1-13. . "Meditación de Occidente". Realidad: revista de ideas, vol. 7, 1948. 
Ruvituso, Clara. Diálogos existenciales: la filosofia alemana en la Argentina peronista (1946-1955). Iberoamericana ; Vervuert, 2015.

Taborda, Saúl, et al. "Frente de Afirmación del Nuevo Orden Espiritual”. Revista Jurídica y de Ciencias Sociales, vol. II, núm. 1, 1932, p. 106. . La crisis espiritual y el ideario argentino. Instituto Social de la Universidad Nacional del Litoral, 1934. . Reflexiones sobre el ideal político de América. [s.n.], 1918.

Terán, Oscar. Historia de las ideas en la Argentina: Diez lecciones iniciales, 1810-1980. Siglo Veintiuno, 2008.

Stabb, Martin S. América Latina En Busca de Una Identidad Modelos Del Ensayo Ideológico Hispanoamericano, 1890-1960. Monte Avila, 1969.

Vázquez, Juan Adolfo, et al., editores. Antología filosófica argentina del siglo XX. Eudeba, 1965.

Weinberg, Liliana, editor. Estrategias del pensar: ensayo y prosa de ideas en América Latina siglo xx. Universidad Autónoma de México, 2010.

Zuleta Alvarez, Enrique. El nacionalismo argentino. La Bastilla, 1975. 